\title{
Is the integration of pop art an important factor in giving birth to the art market?
}

\author{
Yao Wang \\ School of Fine Art Yunnan Normal University \\ 772967536@qq.com
}

Keywords: Pop art; Andy Warhol; Popular art; Art market

\begin{abstract}
Pop art is a new style of art. Its characteristics are popular, popular and mass production, and the characteristics of the art market are to serve the masses and retail a large amount of cheap daily necessities produced in mass production. Pop art is closely related to the supermarket. Andy Warhol is the leader of pop art. He uses supermarket goods as a repeat arrangement, a neat and repetitive arrangement, a brilliant color, and an excellent pop art. The biggest common feature of pop art works and supermarkets is duplication. Similarly, the business philosophy of the market is also to serve the public, and finally the art has become commercialized, and has created a huge art market.
\end{abstract}

\section{波普艺术的融入是否是催生艺术市场的重要因素? \\ 作者 王尧 \\ 云南师范大学美术学院设计系, 昆明市 云南省 中国}

关键词：波普艺术；安迪·沃霍尔；大众艺术；艺术市场

中文摘要. 波普艺术是一种新型的艺术风格, 它的特征是大众化、通俗化、批量生产的; 而艺 术市场的特征是为大众服务, 零售大量批量生产的廉价的日常生活用品。波普艺术与超市有 着千丝万缕的联系。安迪 - 沃霍尔是波普艺术的领袖, 他用超级市场商品的摆放同样是重复 排列, 整齐而重复的排列, 艳丽的色彩, 远观亦是一幅优秀的波普艺术作品。波普艺术作品 与超市最大的共同点是重复。同样, 市场的经营理念同样是为大众服务, 艺术最终走向商品 化，并催生了巨大的艺术市场。

\section{1 大众审美的产物一一波普艺术}

\section{1 波普艺术}

波普艺术是20世纪的流行艺术（popular art），也是流行艺术的简称，被称之为新写实主 义艺术，因为波普艺术（Pop ART）的POP是 “流行的、时尚的”（popular）的英文缩写。 它也是20世纪流行文化的代表之一。作为20世纪后现代艺术之一, 波普艺术影响力最广泛、 传播力度最大, 最能广泛普及的一种艺术形式。所谓波普艺术, 是指艺术主体把所有的额外 负担客观存在物作为艺术载体, 并赋予其价值与内涵, 以传输给观赏者最大众化、最普及化 的艺术形式，以期与观赏者精神上引起共鸣。

\section{2 波普艺术的特点}

波普艺术在产品包装和普及上有着举足轻重的作用, 它将市场中的商业元素用于艺术中。 因此, 当代的艺术流行, 正是由于在波普艺术的助力下得到了长足的发展, 同时波普艺术在 市场中也取得了巨大的成功，由于当代的传媒已趋向大众化、商业化，波普艺术成为 $20 世$ 纪 
最能广为流传的艺术形式, 至此波普艺术形成了一股吹向大众生活的流行之风。波普艺术是 在美国现代文化的影响下催生出的艺术形式，是一种世界范围的文化运动，多以上层社会的 典型形象或戏剧中的偶然事件作为主题。它反映了战后重建社会中的新青年一代对于文化的 价值取向, 力求体现自我价值，追求新事物的心理状态。

\section{3 美国最具代表性的波普艺术家一一安迪・沃霍尔（Andy Warhol）}

安迪·沃霍尔 (Andy Warhol,1928.8.6-1987.2.22) 是20世纪最有影响力的艺术家之一，他 提倡波普艺术, 同时也是波普艺术的领袖人物, 他对波普艺术的影响巨大。他敢于创新, 例 如运用凸版印刷、橡皮或木料拓印、金䈃技术、照片投影等各种手段技法。他的波普艺术也 影响了上世纪80年代至 90 年代的中国艺术, 在这期间, 中国艺术也形成了一阵波普风, 有些 学者还将政治人物画像和 “文革”海报称之为中国的政治波普艺术。

\section{2 商业选择了波普还是波普选择了商品}

\section{1 商品和波普}

安迪 (Andy Warhol) 看明白了商品。商品的本质是复制。而不是价钱, 商品价格的产生, 是建立在你想占为己有的时候。商品也就是物品, 物品是有使用价值的, 而没有具体的内涵, 物品设计的再精巧还是逃不过商品的性质范围。物品谁都可以看得懂。但我们有时也会看不 懂物品, 而且大部分人都看不懂或不理解当代艺术品。原因是大部分人都不理解 “这个东西” 的价值何在? 艺术中的感情是难能可贵的, 之所以被称之为艺术品, 是在这个物品中附着着 艺术家的感情, 是情感传达的媒介。那个时候很多物品的出现让人眼花缭乱。而安迪的职业 是画广告画的。这种最基础的手段对一个艺术家的影响可见一斑。安迪作的作品大部分都是 美式广告画。物品复制忽然变得如此得心应手, 对艺术的影响巨大, 同时代很多的工匠和艺 术家面临的将是失业。

物品的复制影响着人类的生活。复制标志着印刷术的开启, 也是真正的信息开始广为流传 的时代, 标志着现代西方走向强大。而不是蒸汽机。纵观历史可以发现, 中国的织布机和水 车技术是要早于西方的蒸汽机的。可是现在的3D打印机也上市了。

爱马仕这个国际时尚品牌，如果没人用它了，它也不会成为现代生活中的时尚。时尚除了 美的特点, 还有缺稀性。我们谈论安迪崇尚时尚, 虽然他在世界范围的影响很大, 但却不知 道如何谈论他。能认识到这个问题的人很少。精神是神圣的, 但任何精神都是被赋予的, 不 是原本就有的。

安迪（Andy Warhol）： “你想想, 百货商店就是一个博物馆”。从中可以看出他的独特 的审美眼光，他不仅是一个商业艺术家，同时还是个哲学家。

\section{2 审什么美?}

审美在很早以前是欣赏。但美这个词很宽泛。艺术的起源是人们的感知, 表达是情感宣 泄的需要。郑也夫说, 开始的时候语言简单, 美这个词以一当十, 我们就把一切舒服的统称 为美。但是一个搞美术的人一定会重视美, 在意美的成因。美术的递进思维过程是很有趣的。 先有感知, 随后有了观察, 然后开始表现, 就算是写实描绘也是具有抽象特征的, 写意就更 抽象了: 在印象派那里产生了反思, 一开始反思眼睛这个观察器官, 这个折射元素很有可能 是骗人的, 梵高和康定斯基也同时感觉是这样, “阳光穿过你却改变了自己的方向” ; 变化 的太快了, 安迪一看, 前人的视网膜已经破了, 索性就把这个思考扎入人脑里。让人一时适 应不了。这就用自己的生命检验出了答案。美是必然还是偶然? 都不是。葱要和酱作伴, 没人 拿着葱也是酱。而人的伴侣就是感觉器官。非常客观。美赤身裸体被逼到了墙角, 无所遁形。 此时, 物品已被扒光, 但它依然变化着, 这种变化, 就是思维的变化。 


\section{3 波普艺术和商业}

\section{1 艺术向商业化、市场化迈进}

使用波普艺术语言的创作流行在世界各地，波普艺术已经成为世界性语言。这种世界性 语言的形成依靠于社会消费, 成为全球化文化现象, 只要社会有消费的地方, 波普艺术就有 其生存的土壤。艺术品进入市场, 它就已经属于流通的“商品”, 商品有使用价值, 它能满足人 类的某种需求，或者制造某种效应。既然它流通，并且有一定的“交换价值”，那么它就是有用 的（使用价值）“商品”，而价格，指的是不是“使用价值”（这个价值无法用金钱估量），是商 品的价值的体现。

商品的价格只能体现相对的价值，而且还涉及到供求关系（买方市场和卖方市场）。同 时，价格的波动其实跟“价值规律”是一样的，只要了解了艺术品的使用价值，供求关系，就能 理解为什么艺术品能买这么贵。当然, 重点在于, 艺术品的使用价值到底是什么。

\section{2 艺术导向 “非传统” 形式}

艺术品跟生活必需品不一样，它是一种精神奢侈品，价值不是靠什么“生产条件”，“劳动 时间”, “社会平均劳动熟练程度”之类的东西来衡量的。比如 300 年前的画, 如说画技, 工艺, 理论水平, 美学, 现在的画肯定比旧画要高级, 但艺术价值不仅仅是看艺术史的先进性, 还 要看所处的历史地位, 和美学意义。劳动强度也不是衡量艺术价值的标准, 劳动强度也不仅 仅是手工性, 甚至跟专业 (科班出身) 与否都没关系, 还有很多艺术家是版画出身, 建筑出 身, 设计师（培根20出头时就是个室内装饰设计师）。更有一些街头艺术家像基斯·哈林, 巴 斯奎特这种奇才都是天赋异禀的。唐宋元, 到明清, 国画都是统治地位, 鸦片战争, 八国联 军侵华后, 价格骤降, 损坏和流失异常严重。经济对价格的影响更是明显, 金融危机的时候, 艺术市场同样不可避免。

\section{3 市场 “炒作” 催生艺术价值商业模式的建立}

决定艺术品价格的要素, 最主要的, 当然还是艺术水准, 学术质量了, 这是个基本要求。 而参与市场运作后, 市场知名度高的往往跟占便宜。一般作品价格高的艺术家一直以来都有 很高的威望, 学术上已有广泛认同, 而且大多已谢世。现在市场上投资的对象大多集中在印 象派, 后印象派, 巴黎画派和 20 世纪现代艺术, 战后艺术的作品, 这也是为什么佳士得, 苏 富比会专门以年代分出几个部门来进行拍卖。这些艺术家如梵高, 毕加索, 夏加尔, 贾斯帕·约 翰, 贾科梅蒂等都是艺术史上公认的大师, 声誉很好, 尤其安迪这种消费主义的作品更是再 适合不过。同一艺术家的不同题材, 不同尺寸, 创作年代 (时代背景), 作品的传承 (先后由 谁收藏，，存世量，在艺术家创作生涯中的地位，购买者的能力，购买者的投资观念，历史 文化影响（民族情结，文化差异等）市场趋势通货膨胀等也会影响价格。安迪·沃霍尔试图打 破“越是稀缺的作品价值越高”的惯常说法, 他的作品就是“重复、重复再重复”, 虽然不可避免 的会被”稀缺“的概念困住，但因为波普艺术的流通量，事实上他已经做到了。他在半个世纪 前做的事, 现在大家才渐渐理解, 而市场则从他去世后才开始真正的运作。在越来越多承认 之后, 价值越来越高, 从21世纪初就已经开始了。

“赚钱就是艺术，工作就是艺术，好的生意就是最好的艺术”，安迪・沃霍尔曾说到。

\section{4 结语}

波普艺术充满争议，矛盾自然也充斥其间，存在与商业又独立与世界，随意而为又捉摸 不定, 它的商业价值远超于其艺术价值, 肤浅的华丽与神秘的暖昧共存, 象征意义也远超于 之前的艺术样式。 
在沃霍尔之后，毕加索是最受欢迎的艺术家，2015年共售出 2820 件作品，成交额达4.487 亿美元。其中就包括了他的情人多拉 - 玛尔（Dora Maar）的肖像，在2015年5月份拍得2260万 美元，还有《The Rescue》，在2014年5月售得3150万美元。

在毕加索之后的则是弗朗西斯 - 培根, 虽然他只有 114 件作品售出, 但是成交额达到了 3.06 亿美元。仅《约翰・爱德华兹三联画作》一件作品就拍得4740万美元。

在排名前 10 的艺术家中，只有一位在世艺术家，他就是格哈德・里希特，是2015年成交 额第四的艺术家, 作品拍卖成交额达 2.946 亿美元。第五则是马克 - 罗斯科, 他的15幅画作售 得 2.81 亿美元。可以看出, 消费社会有利也有弊, 一方面, 人类社会需要建立以市场经济为基 础的商品社会; 另一方面, 人们也要防止消费主义使文化的扁平化、肤浅化和商品化。

艺术越来越疯狂，波普艺术市场走向的路，不得而知！

\section{References}

[1] Wang S Z, world history of modern design [M]. Beijing: China Youth Publishing House,

[2] Ma Y J, postmodernist art 20 lecture [M]. Shanghai: Shanghai Academy of Social Sciences Press.

[3] Tillman Osteward, pop art [M].translated. Hongkong: Tower publisher, 1999.

[4] Zhu Q S, this is the [J]. Art of modern, Art, 2001 (4). 